\section{Behandlung des metastasierten Prostatakarzinoms}

\author{
P. Albers ${ }^{1}$ \\ ${ }^{1}$ Direktor der Klinik für Urologie, Universitätsklinikum Düsseldorf
}

Das Prostatakarzinom ist der häufigste Tumor des Mannes: Bei seit Jahren steigender Inzidenz sterben in Deutschland pro Jahr trotz einer insgesamt relativ geringen Mortalität über 12000 Männer an diesem Tumor. Durch sein Metastasierungsmuster und die langwierigen Krankheitsphasen verursacht dieser Tumor zusätzlich enorm hohe Behandlungskosten. Nach der Entdeckung der Hormonabhängigkeit des Tumors in den 40er-Jahren des letzten Jahrhunderts (Nobelpreis für Paul Huggins) ist bisher die hormonablative Therapie des androgenabhängigen Karzinoms Standardtherapie bei Patienten mit metastasierter Erkrankung. Bei Vorliegen von Knochenmetastasen leben die Patienten in Abhängigkeit vom Wachstumsmuster 3-5 Jahre, bis der Tumor nicht mehr auf die Hormontherapie anspricht. Bis vor etwa 12 Jahren gab es nach der Hormontherapie keine Systemtherapie, die einen nachweisbaren Überlebensvorteil generieren konnte. Symptomatische Patienten mit kastrationsresistentem Prostatakarzinom lebten in den 90er-Jahren unter einer Therapie mit z.B. Mitoxantron im Mittel etwa $1 \mathrm{Jahr}$ und diese Systemtherapie zeigte keinen signifikanten Unterschied zur Prednisolon-Gabe allein (Tannock JCO 1996). Mit der Einführung von Docetaxel, einem Taxan, in die Systemtherapie des kastrationsresistenten Prostatakarzinoms im Jahr 2004 änderte sich diese Perspektive dramatisch. Docetaxel zeigte in einer großen randomisierten Studie zwar nur einen Überlebensvorteil von median 2,4 Monaten, aber das Gesamtüberleben unter Docetaxel erreichte nunmehr fast 19 Monate (Tannock NEJM 2004). Die Weiterentwicklung der klassischen Chemotherapie beim kastrationsresistenten Prostatakarzinom gelang letztlich nicht, und der letzte Versuch, Docetaxel-Resistenzen mit Cabacitaxel zu behandeln und dieses Präparat dann in der Erstlinie einzuführen, scheiterte mit der gerade auf dem ASCO 2016 vorgestellten 1st-Line-Studie Docetaxel vs. Cabacitaxel (Firstana). Andererseits wurde mit Abirateron eine neue antiandrogene Therapie zur Therapie des Prostatakarzinoms eingeführt und dieses Präparat zeigte in einer großen randomisierten Studie gegen Prednisolon und Placebo nach Versagen der Docetaxel-Therapie ein Gesamtüberleben von zusätzlichen 15 Monaten (De Bono NEJM 2011; Fizazi Lancet Oncology 2012). Daraufhin wurde die neue Hor- montherapie auch in der Erstlinie geprüft und konnte in einer weiteren großen randomisierten Studie ein Gesamtüberleben von etwa 2 Jahren erreichen (Ryan NEJM 2013). Diese Therapie war damit im Vergleich zu den bisherigen Daten mit Docetaxel erfolgreicher und gleichzeitig nebenwirkungsärmer. Parallel erfolgte die Entwicklung anderer neuer Antiandrogene (z.B. Enzalutamid) und weiterer Medikamente, die z.T. andere Wirkmechanismen betrafen (z.B. DNA-Repair durch Olaparib und MET-Inhibition durch Cabozantinib). Hier stehen in den nächsten Jahren sicher weitere Entwicklungen an. Insbesondere wird die Neu-Entwicklung von Biomarkern wichtig werden. Erste Ergebnisse der Analyse von ARV7-Mutationen und Korrelation zum Therapieansprechen sind ebenso interessant wie das Therapiemonitoring in der Systemtherapie durch zirkulierende Tumorzellen oder freie DNA (Andronarakis NEJM 2015). Zielgerichtete Therapieansätze wie bei anderen metastasierten Tumoren stehen beim Prostatakarzinom unmittelbar bevor und werden die Therapieerfolge in Bälde weiter verbessern. Insgesamt können im Jahr 2016 Patienten mit einem metastasierten und kastrationsresistenten Prostatakarzinom nunmehr ein Überleben von häufig 3-4 Jahren erwarten. Die Lebensqualität mit den neu entwickelten Präparaten ist dabei gut. Einziges Problem bleibt die insgesamt teure lebenslange Therapie von Patienten mit metastasierten Prostatakarzinomen. Hier müssen gesundheits-ökonomische Untersuchungen und gesellschaftliche Diskussionen zeigen, wie die weitere Entwicklung von wirksamen Präparaten gestaltet werden soll.

Interessenkonflikt: Honorare für Vorträge und Beratertätigkeit: Sanofi, BMS und Roche

\section{Bibliografie}

DOI http://dx.doi.org/10.1055/s-0042-114455

Drug Res 2016; 66, Suppl. 1: S35-S35

(c) Georg Thieme Verlag KG Stuttgart · New York . ISSN 2194-9379

\section{Korrespondenzadresse \\ Univ.-Prof. Dr. Peter Albers \\ Direktor der Klinik für Urologie Universitätsklinikum Düsseldorf \\ Moorenstraße 5 \\ 40225 Düsseldorf \\ urologie@uni-duesseldorf.de}

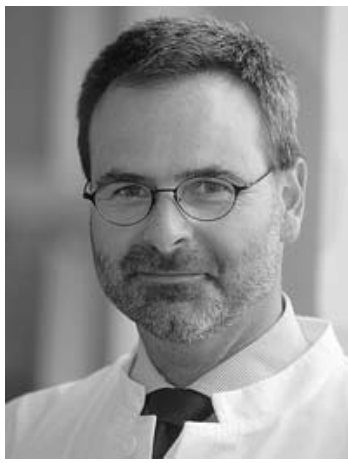

